\title{
The interest of gait markers in the identification of subgroups among fibromyalgia patients
}

\author{
Bernard Auvinet ${ }^{1 *}$, Denis Chaleil ${ }^{2}$, Jean Cabane ${ }^{3}$, Anne Dumolard ${ }^{4}$, Pierre Hatron ${ }^{5}$, Robert Juvin ${ }^{4}$, \\ Michel Lanteri-Minet ${ }^{6}$, Yves Mainguy ${ }^{7}$, Laurence Negre-Pages ${ }^{8}$, Fabien Pillard ${ }^{9}$, Daniel Riviere ${ }^{9}$ and \\ Yves-Michel Maugars ${ }^{10}$
}

\begin{abstract}
Background: Fibromyalgia (FM) is a heterogeneous syndrome and its classification into subgroups calls for broadbased discussion. FM subgrouping, which aims to adapt treatment according to different subgroups, relies in part, on psychological and cognitive dysfunctions. Since motor control of gait is closely related to cognitive function, we hypothesized that gait markers could be of interest in the identification of FM patients' subgroups. This controlled study aimed at characterizing gait disorders in FM, and subgrouping FM patients according to gait markers such as stride frequency (SF), stride regularity (SR), and cranio-caudal power (CCP) which measures kinesia.

Methods: A multicentre, observational open trial enrolled patients with primary FM (44.1 $\pm 8.1 \mathrm{y}$ ), and matched controls (44.1 \pm 7.3 y). Outcome measurements and gait analyses were available for 52 pairs. A 3-step statistical analysis was carried out. A preliminary single blind analysis using k-means cluster was performed as an initial validation of gait markers. Then in order to quantify FM patients according to psychometric and gait variables an open descriptive analysis comparing patients and controls were made, and correlations between gait variables and main outcomes were calculated. Finally using cluster analysis, we described subgroups for each gait variable and looked for significant differences in self-reported assessments.
\end{abstract}

Results: SF was the most discriminating gait variable (73\% of patients and controls). SF, SR, and CCP were different between patients and controls. There was a non-significant association between SF, FIQ and physical components from Short-Form $36(p=0.06)$. SR was correlated to FIQ $(p=0.01)$ and catastrophizing $(p=0.05)$ while CCP was correlated to pain $(p=0.01)$. The SF cluster identified 3 subgroups with a particular one characterized by normal SF, low pain, high activity and hyperkinesia. The SR cluster identified 2 distinct subgroups: the one with a reduced SR was distinguished by high FIQ, poor coping and altered affective status.

Conclusion: Gait analysis may provide additional information in the identification of subgroups among fibromyalgia patients. Gait analysis provided relevant information about physical and cognitive status, and pain behavior. Further studies are needed to better understand gait analysis implications in FM.

\section{Background}

In clinical trials and observational research studies, fibromyalgia (FM) is usually diagnosed according to American College of Rheumatology (ACR) 1990 criteria [1]. However, patients fulfilling the ACR classification criteria for FM do not constitute a homogeneous group and the classification of FM into different subgroups calls for broad-based discussion. Strazt et al [2] sought

\footnotetext{
*Correspondence: bernard.auvinet@gmail.com

'Locomotion Research Unit, Laval Hospital, 53015 Laval, France

Full list of author information is available at the end of the article
}

to identify different FM subgroups by distinguishing between FM with and without depression. Turk et al [3] showed that subgroups identified by cluster analysis, based on the Multidimensional Pain Inventory (MPI), could be applicable to FM patients; this subgrouping was based on psychosocial and cognitive characteristics. Giesecke et al [4] showed that the combination of these two features and pain sensitivity indices best distinguished subgroups of FM patients. Using the MPI, Thieme et al [5] identified three subgroups of FM patients on the basis of anxiety and psychiatric
Ciomed Central 
comorbidities. On the basis of the associated clinical signs and symptoms, Müller [6] found that primary FM can be divided into four subtypes: sensitivity to pain, comorbid pain-related depression, concomitant depression, and FM due to somatization. Recently, De Souza et al [7] featured two distinct subgroups using the Fibromyalgia Impact Questionnaire (FIQ): one was characterized by low anxiety levels, depression and morning tiredness while the other was characterized by elevated pain levels, fatigue, morning tiredness, stiffness, and depressive symptoms. Using the Medical Outcomes study 36-item Short Form Health Survey (SF-36), Oswald et al [8] detected two subgroups: the first one demonstrated psychological dysfunction, whilst the second achieved normal psychological scores. In a large survey, Wilson et al. [9] identified 4 subgroups of FMS patients by means of a cluster analysis based on 3 symptom factor scores: musculoskeletal symptoms, other physical symptoms, and cognitive/psychological symptoms. All mentioned studies illustrate the great interest of clinicians, in daily practice, in recognizing homogenous subgroups in FM to provide guidance for treatment decisions. Furthermore, research is ongoing to assess FM severity and subgrouping FM patients by means of quantitative measurements. More recently, Aparicio [10] found that handgrip was reduced in women with FM $(\mathrm{p}<.001)$, and inversely related to FM severity and symtomatology.

Gait analysis shows a growing interest in the neuropsychological domain, in particular with regard to cognition and dementia [11], as well as anxiety and depression [12]. The importance of cognition for gait became evident with the observation that frail or cognitively impaired elderly people could no longer walk while performing a secondary task such as talking [13].

Gait has to be considered as a cognitive function and not as an automated motor task [14]. Walking at a selfselected speed requires cognitive resources, particularly executive function and attention [15]. Given that FM patients have cognitive function impairment that could mimic about 20 years of ageing [16] we hypothesized that gait could be impaired in FM patients. Affect also has to be taken into account; indeed, depression and anxiety have a negative influence on gait, possibly by reducing attention to gait control [17].

In a preliminary study, we showed that gait impairment in FM patients affects walking speed and walkingderived markers such as stride frequency (SF), stride regularity (SR), and cranio-caudal power (CCP) which measures the kinesia of the movement according to the cranial-caudal axis [18]. Furthermore, Heredia Jiménez [19] found a significant difference between FM women and control groups as regard spatial-temporal parameters of gait. In addition, correlations were found between FIQ and spatial-temporal parameters of gait in FM [19]. Therefore, we decided to undergo a case-control observational study on the interaction between FM and gait in primary non-depressed FM patients without any neurological treatment.

Our study aimed at characterizing gait disorders in primary FM patients, looking for correlations between gait markers and main FM features, and subgrouping FM patients according to gait markers.

\section{Methods}

\section{Study Design}

This multicenter study was an exploratory, observational open trial enrolling outpatients with primary FM and healthy control subjects matched for age, height, and weight. The one-to-one matching was realized by each participating center. Ethics approval for this study was obtained from the Human Ethics Committee of Nantes University. All participants provided informed written consent according to Helsinki declaration.

\section{Study selection criteria}

Healthy control subjects and patients were women aged 20 - 55 years able to walk 40 meters without assistance. Healthy control subjects, if possible without any concomitant medications, underwent a complete physical examination. Patients with primary FM were recruited according to ACR criteria. All of them had achieved an average pain visual analogue score (VAS) of at least 40 on a scale of $0-100 \mathrm{~mm}$, over the past week. They presented non-major depression according to the MiniInternational Neuropsychiatric Interview (MINI) [20].

Patients and controls with any of the following conditions were excluded from the study: current or past cardiovascular, pulmonary, neurological, rheumatologic, endocrine, digestive or renal diseases; current systemic infection; active cancer; and any history of/or existing musculoskeletal disorders that could induce walking disability such as obesity (BMI $>30 \mathrm{~kg} / \mathrm{m}^{2}$ ), pregnant or nursing women, or current psychiatric illnesses.

A routine work-up was performed at a central laboratory for all FM patients. Normal results constituted one of the study inclusion criteria.

\section{Study procedure}

Data from a previous study [18] were used to calculate the study power and the sample size. Gait markers differed considerably and were of clinical value in 14 FM patients and 14 matched controls. Thus a sample size of 22 subjects in each group would provide statistical power of $>0.95$ with an alpha level of 0.05 to assess physical functions by gait analysis in FM patients and matched control subjects. No information was available for the subgrouping of patients by gait analysis. Based 
on this data, we planned to include 60 patients and 60 controls. Study participants were screened/selected at the time of a first visit (V1). Baseline assessments and gait tests were realized at the second visit (V2), which took place between one and four weeks later, depending on the wash-out period needed for discontinuing central nervous system (CNS) active drugs such as antidepressants, antiepileptics, mood stabilizers, centrally acting muscle relaxants, hypnotics, and analgesics type II or III. Patients' consent for drugs withdrawal was included in their informed written consent. When patients experienced an exacerbation of their FM pain severe enough to require additional analgesia, paracetamol was allowed as a rescue analgesic. Likewise, low doses of benzodiazepines were allowed in case of anxiety.

\section{Assessment tools}

At V1, demographic data, exercise status, lifestyle, physical examination, vital signs and patients' biological tests were obtained. In addition, three psychological assessments were carried out: the MINI, the State-Trait Anxiety Inventory (STAI) and the Beck Depression Inventory (BDI). Only certain parts of the MINI questionnaire were used, particularly those exploring major depression, generalized anxiety, panic disorder, agoraphobia, social phobia, obsessive compulsive disorder, post-traumatic syndrome, alcohol and substances abuse and psychotic disorders [20]. The STAI was used to assess state and traits of anxiety [21]. The BDI was used to quantitatively assess depression symptoms of patients [22]. Pain during the previous week was evaluated at V1 and V2 by means of the 100-mm VAS pain score. VAS at V1 and V2 were similar so we referred to VAS at V1. Baseline assessments included the Short Form McGill Pain Questionnaire (SFMPQ) [23]. The Coping Strategies Questionnaire (CSQ) was used to assess patient's strategies to cope with chronic pain, and the efficacy of these strategies in controlling pain [24]. The CSQ assessed the use of 6 cognitive coping strategies (diverting attention, reinterpreting pain sensations, coping self-statements, ignoring pain sensations, praying or hoping, catastrophizing) and 1 behavioral coping strategy (increasing activity level). Fatigue intensity was measured by the Chalder Fatigue Scale (CFS) [25]. Sleep quality and disturbances were assessed by the Pittsburg Sleep Quality Index (PSQI) [26]. The SF36 , including both physical component summary (PCS) and mental component summary (MCS), was utilized for the assessment of health status, functional status and quality of life [27]. The FIQ was used to assess the overall symptomatology of FM patients [28].

\section{Gait analysis}

The gait analysis system (Locometrix ${ }^{\mathrm{TM}}$ ) includes an accelerometric sensor, a recording device and a computer program for processing the acceleration signals. The sensor, composed of three accelerometers, is incorporated into a semi-elastic belt, which is fastened around the subject's waist. One accelerometer is aligned with the cranio-caudal axis of the body. A gait test was performed during a stabilized walk at a self-selected speed, which allowed obtaining stabilized gait measurements. Patients and controls wore their usual walking shoes. The duration of gait analysis was long enough to include 19-21 gait cycles.

\section{Gait markers}

The analysis software allowed the characterization of gait by measuring the following markers: walking speed, SF, stride length, right and left step symmetry, $\mathrm{SR}$, and CCP. Walking speed is measured in meter per second $(\mathrm{m} / \mathrm{s})$, and $\mathrm{SF}$ is the number of gait cycles per second $(\mathrm{Hertz}(\mathrm{Hz}))$. Stride length is calculated from the average speed divided by the SF (meter $(\mathrm{m})$ ). Right and left step symmetry on vertical accelerations is an index of overall symmetry (dimensionless). SR quantifies the spatial-temporal similarity between successive gait cycles, which is a measure of stride-to-stride variability (dimensionless). CCP (W/ $\mathrm{kg}$ ) measures the amount of movement (magnitude and frequency) in the cranio-caudal axis and can be considered as a measurement of the kinesia [29].

\section{Statistical analysis}

The primary assessment was the quantitative evaluation of gait markers. A preliminary single blind analysis was performed as an initial validation of gait markers. The statistician had to cluster all participants (patients and controls, identities were coded) into FM patients and control subjects using k-means cluster analysis limited to two clusters. Then the descriptive analysis was performed on each gait marker alone and then by combining different markers in order to improve the sensitivity or specificity i.e. the ability to detect FM or controls. Comparisons between FM patients and control subjects were made in an open statistical analysis. The purpose was to quantify FM patients according to psychometric, self-questionnaire assessment and gait markers. Main outcomes were obtained using all items, which differed significantly between patients and controls without any center effect. In addition, ROC curve analysis of gait markers was performed. Finally, correlations between gait markers and main outcomes were calculated.

Thirdly, using the hierarchical cluster analysis and calculating the Euclidian distances between groups according to Ward, we described subgroups for each gait marker and looked for significant differences in self reported assessments. 


\section{Results}

\section{Population characteristics}

Out of 132 subjects (73 FM patients and 59 controls) eligible to be enrolled at V2, 104 (52 matched pairs) completed the study according to the protocol. Table 1 shows that patients and controls characteristics at V1 were not statistically different except for professional status, concomitant treatment, STAI-trait, and BDI. The STAI-state was not different between patients and controls. No pain was recorded in the control group. Self-report measures at V2 are shown in table 2. The variance analysis showed a center effect for physical functions in FIQ $(P<0.05)$, and reinterpreting pain sensations in CSQ $(P<0.05)$. These 2 items were therefore excluded from the statistical analysis. Gait markers in patients and controls, and variance analysis of the center effect are shown in table 3. Consequently, 3 gait markers were selected for statistical analysis: SF, SR and CCP. ROC curves confirmed the utility of gait markers in the identification of patients (area under the curve for SF, SR and CCP were 0.740 (0.044), $0.678(0.052)$ and $0.690(0.053)$, respectively). The blind cluster analysis showed that SF was the most discriminating marker among patients and controls (38/ $52(73 \%))$.

Table 1 Characteristics of the study population at the time of the screening visit

\begin{tabular}{|c|c|c|c|}
\hline Measurements & $\begin{array}{l}\text { FM patients } \\
\mathrm{n}=52\end{array}$ & $\begin{array}{l}\text { Controls } \\
n=52\end{array}$ & $P$ \\
\hline Age, mean (SD) years & $44.1(8.1)$ & $44.5(7.3)$ & 0.59 \\
\hline Height, mean (SD) cm & $165(5.8)$ & $164(6.4)$ & 0.35 \\
\hline $\mathrm{BMI}$, mean $(\mathrm{SD}) \mathrm{k} / \mathrm{m}^{2}$ & $24.2(4.1)$ & $23.8(4.4)$ & 0.95 \\
\hline Marital Status & & & 0.57 \\
\hline Married, n & 33 & 38 & NS \\
\hline Other situations, n & 19 & 14 & NS \\
\hline Education level & & & 0.97 \\
\hline Primary education, $\mathrm{n}$ & 2 & 2 & NA \\
\hline Secondary education, n & 27 & 26 & 0.98 \\
\hline Higher education, n & 23 & 24 & 0.98 \\
\hline Professional Activity, n & 50 & 32 & $<0.0001$ \\
\hline Physical exercise, n & 32 & 32 & NS \\
\hline Concomitant treatment, $\mathrm{n}$ & 37 & 17 & $<0.0001$ \\
\hline BDI, mean score (SD) & $15.8(8.2)$ & $3.8(3.7)$ & $<0.0001$ \\
\hline STAI-State, mean score (SD) & $37.3(13.6)$ & $29.6(8.4)$ & 0.16 \\
\hline STAI-Trait, mean score (SD) & $44.1(12.6)$ & $35.6(9.0)$ & 0.02 \\
\hline VAS weekly, mean score (SD) & $63.4(21.5)$ & $2.8(7.8)$ & 0.0001 \\
\hline Tender points, mean (SD) & $16.2(2.1)$ & 0 & NA \\
\hline Pain duration, mean (SD) years & $7.3(6.6)$ & 0 & NA \\
\hline
\end{tabular}

NS = not stated; NA = not applicable; BDI = Beck Depression Inventory; STAI = State-Trait Anxiety Inventory; VAS = Visual Analog Scale.

\section{Correlation analysis}

No correlation was found between gait markers and SFMPQ, CFS, PSQI, SF-36. A tendency to correlation was shown between SF-36 PCS and SF $(P=0.06)$. VAS was negatively correlated to CCP $(\mathrm{r}=-0.33, P=0.01)$. The FIQ score was negatively correlated to SR $(\mathrm{r}=-0.34, P$ $=0.01)$, and had a tendency to correlate with SF ( $\mathrm{r}=$ -0.26, $P=0.06$ ). Among CSQ items, only diverting attention and coping statement were positively correlated to SF. Coping self statement and catastrophizing were positively and negatively correlated to SR, respectively (Table 4).

\section{Cluster analyses}

The cluster analysis of SF (Table 5) allowed the identification of 3 distinct subgroups with an Euclidian dissimilarity scale of 17 (F ratio $=76, P=0$ ). The SF mean standard error (SE) for subgroups I $(\mathrm{n}=9)$, II $(\mathrm{n}=24)$ and III $(\mathrm{n}=19)$ was 1.04 (0.01), 0.94 (0.007), and 0.86 (0.008), respectively. No differences were observed for BDI, STAI-trait, SF-MPQ, CSQ, CFS, PSQI, SF-36 MCS subscore and SR. The following differences have been observed between these 3 subgroups: VAS score was significantly lower in subgroup I than in subgroup II $(P$ $=0.01)$. FIQ score was significantly lower in subgroup I compared to subgroups II and III $(P=0.004)$. PCS subscore of SF-36 was significantly higher in subgroup I than group III $(P=0.04)$, and CCP score was significantly higher in subgroup I than the other two subgroups $(P=0.0002)$.

Cluster analysis of SR (Table 6) allowed the identification of 2 distinct subgroups with a dissimilarity scale of 17 (F ratio $=88, P=0)$. The mean $\mathrm{SR} \pm$ standard error for subgroup I $(\mathrm{n}=32)$ was higher than for subgroup II $(n=20) 291 \pm 4$ versus $229 \pm 5$. No difference was observed for VAS, SF-MPQ, CFS PSQI, SF-36, SF and CCP. Patients with low SR were more depressed $(P=$ $0.03)$ and showed more anxiety traits $(P=0.03)$ than patients with normal SR. Furthermore, patients with low SR had reduced coping strategies such as self-statement $(P=0.003)$ as well as greater tendency towards catatrophizing $(P=0.002)$. Finally, low SR was associated with higher FIQ score $(P=0.02)$.

Cluster analysis of CCP values (results not shown) allowed the identification of 3 distinct subgroups with a dissimilarity scale of 17 ( $\mathrm{F}$ ratio $=184, P=0)$. The mean CCP (SE) was higher in subgroup II $(\mathrm{n}=8)$ than subgroups I $(\mathrm{n}=24)$ and III $(\mathrm{n}=20): 5.31$ (0.18) versus $1.41(0.10)$ and $2.92(0.1)$, respectively. On the other hand, the multivaried analysis of these subgroups, based on main outcome measurements, showed no differences between the 3 subgroups, except for VAS that was significantly low in the subgroup II 
Table 2 Self-report measurements at the time of baseline visit

\begin{tabular}{llll}
\hline Measurements & FM patients & Controls & P \\
& $\mathbf{n}=\mathbf{5 2}$ & $\mathbf{n} \mathbf{2}$ & $<0.0001$ \\
\hline VAS weekly, mean score (SD) & $70(18.1)$ & $3.6(9.7)$ & $<0.0001$ \\
SF-MPQ, mean score (SD) & $24.5(9.4)$ & $1.3(3.3)$ & $<0.0001$ \\
FIQ, mean score (SD) & $56.6(15.1)$ & $4.5(8.3)$ & 0.008 \\
CSQ & & & 0.01 \\
$\quad$ Diverting attention, mean score (SD) & $16(7.4)$ & $10(9.3)$ & 0.0009 \\
$\quad$ Reinterpreting pain sensations, mean score (SD) & $9.2(7.7)$ & $5.5(6.4)$ & 0.48 \\
$\quad$ Coping self-statement, mean score (SD) & $24.2(7.1)$ & $16.5(10.6)$ & 0.0002 \\
$\quad$ Ignoring pain sensation, mean score (SD) & $15.6(8.1)$ & $13.5(9.3)$ & $<0.0001$ \\
$\quad$ Praying and hoping, mean score (SD) & $12.1(7.2)$ & $6.8(7.5)$ & 0.09 \\
$\quad$ Catastrophizing, mean score (SD) & $14.5(9.2)$ & $4.6(5.7)$ & $<0.0001$ \\
$\quad$ Increasing activity level, mean score (SD) & $18.8(6)$ & $14.3(9.6)$ & $<0.0001$ \\
CFS, mean score (SD) & $20.8(5.6)$ & $11.3(2.2)$ & $<.3(2.7)$ \\
PSQI, mean score (SD) & $11.3(4.3)$ & & $<0.0001$ \\
SF-36 & & $56(4)$ & 0.002 \\
$\quad$ Physical component summary, mean score (SD) & $34(7.5)$ & $50.9(6.7)$ & \\
$\quad$ Mental component summary, mean Score (SD) & $40.6(10.5)$ & & \\
\hline
\end{tabular}

VAS = Visual Analog Scale; SF-MPQ = Mc Gill Pain Questionnaire; FIQ = Fibromyalgia Impact Questionnaire; CSQ = Coping Strategies Questionnaire; CFS = Chalder Fatigue Scale; PSQI = Pittsburg Sleep Quality Index; SF-36 = Short Form 36 items Medical Outcomes Study Questionnaire

compared to subgroups I and III: 51.9 versus 72.2 and 74.6, respectively, $P=0.006$.

\section{Discussion}

\section{Patients}

Only patients with primary FM were enrolled in the study. Excluded patients were those with secondary fibromyalgia in relation to an underlying disease such as rheumatoid processes that could interfere with gait analysis. All study patients fulfilled the ACR criteria and presented primary FM without major depression based on the MINI questionnaire. However, 3 patients had BDI scores reaching the value of severe depression [30]. This could be due to the fact that total BDI score may give a misleading impression of the nature and degree of affective disturbances in chronic pain, in relation with BDI items assessing physical symptoms [31]. In our study, we found a strong positive correlation between BDI scores and somatic disturbances subscores. According to FIQ values few of our patients have severe FM with a FIQ score over 70 [32]. This finding could be related to the absence of patients with major depression (according to the MINI) as well as obese patients. Depression constitutes one of the items measured by the FIQ. Obesity may contribute to the severity of FM as it reduces physical functioning and increases fatigue [33], which are a part of some FIQ items. However, no significant association between FM symptoms and obesity was found in the same study. On the other hand, obesity was associated with significant gait abnormalities such as lower SF $(p=0.01)$, decreased SR $(p<0.001)$, and reduced CCP $(\mathrm{p}<0.001)$ in comparison with controls (unpublished data). So FM obese patients were excluded in this study.

\section{Gait assessment}

Ambulatory gait analysis has been demonstrated as a reliable method in clinical practice for outpatients, [34]. Such gait analysis systems has been designed for clinicians looking for quantifying gait abnormalities, to grade gait disorder-related disability, and to provide a better pathology's understanding for tailored treatment. This way of thinking about gait analysis was emerged from previous researches conducted in gait analysis

Table 3 Gait markers, center effect, and ROC curve analysis in the study population

\begin{tabular}{llllll}
\hline Gait markers & $\begin{array}{l}\text { Patients } \\
\mathbf{n = 5 2}\end{array}$ & $\begin{array}{l}\text { Controls } \\
\mathbf{n}=\mathbf{5 2}\end{array}$ & $\mathbf{P}$ & Center effect & Area under the ROC curve \\
\hline Walking speed, mean (SD) (m/s) & $1.18(0.19)$ & $1.32(0.17)$ & 0.00007 & $P<0.03$ & ND \\
Stride Frequency, mean (SD) (Hz) & $0.93(0.07)$ & $0.99(0.07)$ & 0.000008 & $P=0.36$ & $0.740(0.044)$ \\
Symmetry, mean (SD) (WD) & $213(39)$ & $227(38)$ & 0.067 & $P<0.03$ & $\mathrm{ND}$ \\
Stride regularity, mean (SD) (WD) & $267(38)$ & $293(39)$ & 0.0007 & $P=0.10$ & $0.678(0.052)$ \\
Cranio-caudal Power, mean (SD) (W/Kg) & $2.59(1.45)$ & $3.63(1.51)$ & 0.0005 & $P=0.12$ & $0.690(0.053)$ \\
\hline
\end{tabular}

$\mathrm{ND}=$ not done; $\mathrm{WD}=$ without dimension 
Table 4 Correlations between gait markers and main outcomes measurements

\begin{tabular}{llll}
\hline Outcomes measurements & Gait markers & & CCP \\
\cline { 2 - 3 } & Stride frequency & Regularity & $r=-0.33, P=0.01$ \\
VAS weekly pain & $r=-0.26, P=0.06$ & $r=-0.34, P=0.01$ & \\
FIQ score & $r=0.42, P=0.002$ & \\
CSQ score & $r=0.28, P=0.04$ & $r=0.31, P=0.03$ \\
Diverting attention & & $r=-0.27, P=0.05$ \\
Coping self-statement & $r=0.27, P=0.06$ & \\
Catastrophizing & & \\
SF-36 (PCS) & & \\
\hline CCP = Cranio-caudal Power; VAS = Visual Analog Scale; FIQ = Fibromyalgia Impact Questionnaire; CSO; Coping Strategies Questionnaire; SF-36 (PCS) $=$ Short Form
\end{tabular}
36 items Medical Outcomes Study Questionnaire- physical component summary

laboratory, including FM patients [35]. Nevertheless, gait analysis has to be considered as a complementary exam. The gait analysis system, gait tests and derived markers have been previously validated [36,37]. Walking at selfselected overground speed is the gold standard of gait analysis [38]. SF expresses basic rhythmic stepping while SR expresses gait unsteadiness according to temporal and dynamic parameters, which is referred to as gait variability [39]. CCP, previously studied in Parkinson's disease (PD), is correlated to motor score and proposed as a measurement of kinesia [29].

\section{Gait markers in fibromyalgia}

In our study, SF revealed to be the best gait marker differentiating FM patients from controls allowing the identification of 3 out of 4 subjects in each group. This finding raises the question of the importance of SF in $\mathrm{FM}$, and its significance in terms of underlying mechanism, contrary to PD patients in whom SF remained unaltered [40]. In addition, SF in our study was correlated to diverting attention and coping self assessment, which are associated with high physical and low psychosocial disability levels [41]. Finally, we found a weak correlation between SF, FIQ and physical component of SF36. These results suggest that SF may be of interest in assessing the physical component of FM.

In our study, SR was strongly correlated to FIQ and to catastrophizing that is a major CSQ item. In FM, catastrophizing is a main cognitive factor, and can prospectively predict high level of pain and depression, and low quality of life [41]. SR measures the unsteadiness of gait, and it has been linked to many neurological diseases such as PD [29], Alzheimer's disease [42], and preclinical stages of dementia [12]. Interestingly, a recent investigation disputed the concept of automatic regulation and suggested that stride time variability is related to specific cognitive processes, namely executive function and attention [39]. Therefore, SR could be suggested as a measurement of cognitive reserve in FM.

$\mathrm{CCP}$, which measures kinesia, was found to be the only correlation in our study between pain and gait analysis. In fact, CCP measurement reflected the fear of pain (kinesiophobia), which is a classic behavior in FM patients leading to a reduction in their movements [43]. This result highlights the significance of gait analysis and pain behavior assessment in chronic painful conditions previously described in patients with lower back pain [44]. Kinesia evaluation by means of CCP measurement could be a promising area of research in the field of pain behavior management.

Gait markers were not correlated to fatigue, sleep disorders or mental component of SF-36. This result has to be discussed according to the gait test that was primarily designed to analyze gait in the basal condition at a self-selected speed. Additional components to the gait test could thus be suggested such as 6 -minute walk test

Table 5 Cluster analysis of stride frequency and variance analysis of main outcomes measurements in fibromyalgia patients

\begin{tabular}{|c|c|c|c|c|c|c|}
\hline & $\begin{array}{l}\text { Subgroup I } \\
\mathrm{n}=9\end{array}$ & $\begin{array}{l}\text { Subgroup II } \\
n=24\end{array}$ & $\begin{array}{l}\text { Subgroup III } \\
\mathrm{n}=19\end{array}$ & Comparison of subgroups & F ratio & $P$ \\
\hline SF, mean $(\mathrm{SE})(\mathrm{Hz})$ & $1.04(0.03)$ & $0.94(0.02)$ & $0.86(0.02)$ & $I,\|\neq\|$ & 76 & 0 \\
\hline VAS weekly, mean score $(0-100)$ & $56.9(12.9)$ & $76.9(7.1)$ & $67.5(8.1)$ & $I \neq \|$ & 4.99 & 0.01 \\
\hline $\mathrm{FIQ}$, mean score $(0-100)$ & $42.0(10.6)$ & $59.6(5.8)$ & $59.8(6.6)$ & $|\neq \| \&|||$ & 6.20 & 0.004 \\
\hline SF-36 (PCS), mean score $(0-100)$ & $39.9(5.8)$ & $33.5(3.0)$ & $32.2(3.4)$ & $I \neq|| \mid$ & 3.35 & 0.04 \\
\hline $\mathrm{CCP}$, mean $(\mathrm{W} / \mathrm{Kg})$ & $4.2(1.0)$ & $2.6(0.5)$ & $1.9(0.6)$ & $|\neq \| \&|||$ & 10.63 & 0.0002 \\
\hline
\end{tabular}

$\mathrm{SF}=$ stride frequency; SE = standard error; VAS = Visual Analog Scale; FIQ = Fibromyalgia Impact Questionnaire; SF-36 (PCS) = Short Form 36 items Medical Outcomes Study Questionnaire - physical component summary; CCP = Cranio-caudal power 
Table 6 Cluster analysis of stride regularity and variance analysis of main outcomes measurements in fibromyalgia patients

\begin{tabular}{lllll}
\hline & $\begin{array}{l}\text { Subgroup I } \\
\mathbf{n}=\mathbf{3 2}\end{array}$ & $\begin{array}{l}\text { Subgroup II } \\
\mathbf{n = 2 0}\end{array}$ & F ratio & P \\
\hline SR, mean (SE) (WD) & $291.3(8.4)$ & $229.3(10.8)$ & 88 & 0 \\
BDI, mean score (0 - 63) & $13.8(2.8)$ & $19.0(3.7)$ & 5.35 & 0.03 \\
STAI-Trait, mean score (20 - 80) & $41.2(4.4)$ & $48.9(5.7)$ & 4.97 & 0.03 \\
CSQ self-statement, mean score (0 - 36) & $26.5(2.4)$ & $20.7(3.1)$ & 9.49 & 0.003 \\
CSQ catastrophizing, mean score (0 - 36) & $11.6(3.1)$ & $19.3(4.0)$ & 10.18 & 0.002 \\
FIQ, mean score (0 - 100) & $52.8(5.2)$ & $62.7(6.8)$ & 5.74 & 0.02 \\
\hline
\end{tabular}

$\mathrm{SR}=$ Stride regularity; $\mathrm{SE}=$ standard error; $\mathrm{WD}=$ without dimension; BDI = Beck Depression Inventory; STAI = State-Trait Anxiety Inventory; CSQ = Coping Strategies Questionnaire; FIQ = Fibromyalgia Impact Questionnaire

or dual task in order to consider fatigue and sleep disorders in FM patients.

\section{Cluster analysis}

The cluster analysis strengthened the clinical significance of each gait marker. Cluster analysis of SF identified an interesting subgroup characterized by a normal $\mathrm{SF}$ associated with low pain level (VAS), reduced overall symptomatology (FIQ), high activity (PCS) and hyperkinesia. The two other subgroups with reduced SF were characterized by high pain level, low activity and hypokinesia.

Cluster analysis confirms the correlations observed in the subgroup with low SR, characterized by reduced self-statement, increasing catastrophizing, and high FIQ. This subgroup was also distinguished by more anxiety and depression. Therefore, one may raise the question of possible overlap between the subgroup with low SR, identified on the basis of gait analysis, and other subgroups distinguished by means of different cluster methods based on anxiety, depression and cognitive features. Consequently, considering the important role of affect and cognition in FM patients, one could suggest SR measurement before initiating cognitive-behavioral therapies in order to adapt treatment approaches to patients' characteristics [45]. Cluster analysis of CCP enabled the identification of a subgroup with low pain level and hyperkinesia. Our hypothesis is that each gait marker is correlated to one of the major traits of FM patients such as pain (CCP), physical activity (SF), and catastrophizing (SR).

\section{Clinical value}

The three main gait markers (SF, SR, CCP) were correlated to some major clinical characteristics of patients (VAS, FIQ score, Coping self statement, Catastrophizing, PCF from SF-36). Such statistical results are needed, but are not sufficient for clinical applications. This point received a first answer through Cluster analysis. Each cluster analysis of gait markers provided distinct subgroups in term of mean value of the gait marker taken into account, but homogenous in term of clinical characteristics. Furthermore a preliminary study showed that the improvement of gait markers is of clinical significance in FM patients after a 12-week rehabilitation and exercise training program [46].

\section{Study limitations}

Our study has some limitations. The presence of a center effect concerning some gait markers such as step length and gait speed, on the one hand, and physical activity subscore of FIQ, on the other hand, did not permit the determination of correlations between these items. The reduced speed in FM $[18,19]$ could be more difficult to interpret because it is the product of SF by SL. The assumption that SL is supraspinally controlled by phasic output from basal ganglia to the supplementary motor area should be discussed, not only according to grading and subgrouping of FM but also according to the disease's underlying mechanisms. Another limitation is the absence of FM patients having major depression. Currently, it is demonstrated that depression is associated with gait deterioration and cognitive impairment [47]. Further studies are required in order to better understand gait disorders in FM patients with depression. As well, the exclusion of obese patients from the study population could be considered as a study limitation, however it is important to take into account that the prevalence of obesity varies greatly between the different studies according to the area of the study. For instance, in North America a prevalence of $47 \%$ was reported [33] while in North Europe the prevalence is only of $10 \%$. [48]. Further limitations are related to sample characteristics. Our study involved only women, since the occurrence of FM in men is fairly limited.

\section{Conclusions}

In conclusion, gait marker measurement, in particular $\mathrm{SF}, \mathrm{SR}$, and $\mathrm{CCP}$, could be of interest in subgrouping 
FM patients, with no major depression. In our study, SF was the most sensitive and specific gait marker for distinguishing FM patients from controls. High SF values characterized a hyperkinetic subgroup of FM patients. So, subgrouping FM patients on the basis of SF measurement could facilitate the prescription of physical activity for patients with normal SF, and incite clinicians to prescribe a slowly progressive physical activity program for those with reduced SF. SR allows the identification of two subgroups, which were significantly different in terms of coping, affective status, and overall symptomatology (FIQ). Reduced SR values characterized a subgroup of FM patients with poor affect and cognitive abilities. SR measurement could therefore help in the orientation of the behavioral therapy in this subgroup of patients. Due to the strong correlation between CCP and pain level, we suggest that CCP measurement could be of interest in the assessment of pain behavior among FM patients. Further studies are needed to assess the interest of gait markers in the identification of subgroups of FM patients.

\section{Acknowledgements}

We are grateful to all the patients and controls who participated in the study. We would like to acknowledge Dr Alaa GHALI for his assistance in the preparation of the English draft of this manuscript.

\section{Author details \\ 'Locomotion Research Unit, Laval Hospital, 53015 Laval, France. ${ }^{2}$ Department of Pharmacy, University Hospital, 49045 Angers, France. ${ }^{3}$ Department of Internal Medicine, St Antoine Hospital, 75012 Paris, France. ${ }^{4}$ Department of Rheumatology, University Hospital, 38043 Grenoble, France. ${ }^{5}$ Department of Internal Medicine, University Hospital, 59000 Lille, France. ${ }^{6}$ Pain Department, University Hospital, 06002 Nice, France. ${ }^{7}$ Pierre Fabre Laboratories, Department of Clinical Research, 31319 Labège Innopole, France. ${ }^{8} \mathrm{LN}$ Pharma, Epidemiology, 31000 Toulouse, France. ${ }^{9}$ Respiratory Functional Explorations, Department of Sport Medicine, University Hospital, 31059 Toulouse, France. ${ }^{10}$ Department of Rheumatology, University Hospital, 44093 Nantes, France.}

\section{Authors' contributions}

BA conceived the idea of the study. BA, YM, YMM conceived the project. YM and LNP wrote the protocol and conducted the coordination of the trial. DC designed and performed the statistical analysis. BA and DC wrote the manuscript. All authors contributed to the study design, provided input into the writing of the protocol, were involved in data collection, provided feedback on drafts of this paper, read, and approved the final manuscript.

\section{Competing interests}

This work was supported in part by a grant from Pierre Fabre Laboratories. B.A. received research support form Pierre Fabre Laboratories. Y.M. is an employee and shareholder of Pierre Fabre Laboratories. L.N.P. received honoraria from Pierre Fabre Laboratories. All other authors have declared no conflicts of interest.

Received: 5 November 2010 Accepted: 11 November 2011

Published: 11 November 2011

\section{References}

1. Wolfe F, Smythe HA, Yunus MB, Bennett RM, Bombardier C, Goldenberg DL, Tugwell P, Campbell SM, Abeles M, Clark P, et al: The American College of Rheumatology 1990 Criteria for the classification of fibromyalgia report of the multicenter criteria committee. Arthritis Rheum 1990, 33:160-72.
2. Stratz T, Schochat T, Färber L, Schweiger C, Müller W: Are there subgroups in fibromyalgia. J Musculoskel Pain 1995, 3(Suppl 1):15.

3. Turk DC, Okifuji A, Sinclair JD, Starz TW: Pain, disability, and physical functioning in subgroups of patients with fibromyalgia. J Rheumatol 1996, 23:1255-62.

4. Giesecke T, Williams DA, Harris RE, Cupps TR, Tian X, Tian TX, Gracely RH, Clauw DJ: Subgrouping of Fibromyalgia Patients on the Basis of Pressure-Pain Thresholds and Psychological Factors. Arthritis Rheum 2003, 10:2916-22.

5. Thieme K, Turk DC, Flor H: Comorbid depression and anxiety in fibromyalgia syndrome: relationship to somatic and psychosocial markers. Psychosom Med 2001, 66:837-44.

6. Müller W, Schneider EM, Stratz T: The classification of fibromyalgia syndrome. Rheumatol Int 2007, 27:1005-10.

7. De Souza JB, Goffaux P, Julien N, Potvin S, Charest J, Marchand S: Fibromyalgia subgroups: profiling distinct subgroups using the Fibromyalgia Impact Questionnaire. A preliminary study. Rheumatol Int 2009, 29:509-15.

8. Oswald J, Salemi S, Michel BA, Sprott H: Use of the Short-Form-36 Health Survey to detect a subgroup of fibromyalgia patients with psychological dysfunction. Clin Rheumathol 2008, 27:919-21.

9. Wilson HD, Robinson JP, Turk DC: Toward the identification of symptom patterns in people with fibromyalgia. Arthritis Rheum 2009, 61:527-34.

10. Aparicio VA, Ortega FB, Heredia JM, Carbonell-Baeza A, Sjöström M, Delgado-Fernandez M: Handgrip Strength Test as a Complement Tool in the Assessment of Fibromyalgia Severity in Women. Arch Phys Med Rehabil 2011, 92:83-88.

11. Verghese J, Wang C, Lipton RB, Holtzer R, Xue X: Quantitative gait dysfunction and risk of cognitive decline and dementia. J Neurol Neurosurg Psychiatry 2007, 78:929-35.

12. Snijders AH, Van de Warrenburg BP, Giladi N, Bloem BR: Neurological gait disorders in elderly people: clinical approach and classification. Lancet neurol 2007, 6:63-74.

13. Lundin-Olsson L, Nyberg L, Gustafson Y: "Stops walking when talking" as a predictor of falls in elderly people. Lancet 1997, 349:617.

14. Hausdorff JM, Yogev G, Springer S, Simon ES, Giladi N: Walking is more like catching than tapping: gait in the elderly as a complex cognitive task. Exp Brain Res 2005, 164:541-48.

15. Yogev-Seligmann G, Hausdorff JM, Giladi N: The role of executive function and attention in gait. Mov Disord 2008, 23:329-42.

16. Glass JM: Fibromyalgia and cognition. J Clin Psychiatry 2008, 69:20-4.

17. Hausdorff JM, Peng CK, Goldberger AL, Stoll AL: Gait unsteadiness and fall risk in two affective disorders: a preliminary study. BMC Psychiatry 2004, 4:39.

18. Auvinet B, Bileckot R, Alix AS, Chaleil D, Barrey E: Gait disorders in patients with fibromyalgia. Joint Bone Spine 2006, 73:543-6.

19. Heredia Jiménez JM, Aparicio Garcia-Molina VA, Porres Foulquie JM, Delgado Fernández M, Soto Hermono VM: Spatial-temporal parameters of gait in women with fibromyalgia. Clin Rheumatol 2009, 28:595-8.

20. Sheehan DV, Lecrubier $Y$, Sheehan $\mathrm{KH}$, Amorim P, Janavs J, Weiller E, Hergueta T, Baker R, Dunbar GC: The mini-international neuropsychiatric interview (MINI). The development and validation of a structured diagnostic psychiatric interview for DSMIV and ICD10. J Clin Psychiatry 1998, 59:22-3.

21. Spielberger CD, Gorsuch RL, Lushene RE: Manual for the State-Trait Anxiety Inventory. Palo Alto CA: Consulting Psychologists Press; 1983.

22. Beck AT, Ward CH, Mendelson M, Mock J, Erbaugh J: An inventory for measuring depression. Arch Gen psychiatry 1961, 4:561-71.

23. Wolfe F, Anderson J, Harkness D, Bennett RM, Caro XJ, Goldenberg DL, Russell IJ, Yunus MB: Health status and disease severity in fibromyalgia: results of a six-centre longitudinal study. Arthritis Rheum 1997, 40:1571-9.

24. Rosenstiel AK, Keefe FJ: The use of coping strategies in chronic low back pain patients: relationship to patient characteristics and current adjustment. Pain 1983, 17:33-44.

25. Chalder T, Berelowitz G, Pawlikowska T, Watts L, Wessely S, Wright D, Wallace EP: Development of a fatigue scale. J Psychosom Res 1993, 37:147-53.

26. Buysse DJ, Reynolds CF, Monk SR, Kupfer DJ: The Pittsburg Sleep Quality Index: A new instrument for psychiatric practice and research. Psychiatry Research 1989, 28:193-213. 
27. Ware J, Kosinski M, Dewey J: How to score version 2 of the SF-36 Health Survey (Standard and acute forms). Lincoln, Rl: Quality Metric; 32000.

28. Burckhardt CS, Clark SR, Bennett RM: The fibromyalgia impact questionnaire: development and validation. J Rheumatol 1991, 18:728-33.

29. Paquet JM, Auvinet B, Chaleil D, Barrey E: Analysis of gait disorders in Parkinson's disease assessed with an acceloremeter. Rev Neurol (Paris) 2003, 159:766-9.

30. Beck AT, Steer RA, Carbin MG: Psychometric properties of the Beck Depression Inventory: Twenty-five years of evaluation. Clin Psychol Rev 1988, 77-100.

31. Williams AC, Richardson $\mathrm{PH}$ : What does the BDI measure in chronic pain? Pain 1993, 55:259-66.

32. Bennett R: The Fibromyalgia Impact Questionnaire (FIQ): a review of its development, current version, operating characteristics and uses. Clin Exp Rheumatol 2005, 23:S154-62.

33. Okifuji A, Donaldson GW, Barck L, Fine PG: Relationship Between Fibromyalgia and Obesity in Pain, Function, Mood, and Sleep. The Journal of Pain 2010, 11:1329-1337.

34. Montero-Odasso M, Casas A, Hansen KT, Bilski P, Gutmanis I, Wells JL, Borrie MJ: Quantitative gait analysis under dual-task in older people with mild cognitive impairment: a reliability study. Journal of Neuro Engineering and Rehabilitation 2009, 6:35.

35. Tiidus PM, Pierrynowski MR, Dawson KA: Influence of moderate training on gait and work capacity of fibromyalgia patients: a preliminary field study. J Sports Sci Med 2002, 4:122-7.

36. Auvinet B, Chaleil D, Barrey E: Accelerometric Gait Analysis for Use in Hospital Outpatients. Rev Rhum [Engl Ed] 1999, 66:389-397.

37. Auvinet B, Berrut G, Touzard C, Moutel L, Collet N, Chaleil D, Barrey E: Reference data for normal subjects obtained with an accelerometric device. Gait and Posture 2002, 16:124-34.

38. Dubost V, Annweiler C, Aminian K, Najafi B, Herrmann FR, Beauchet $O$ : Stride-to-stride variability while enumerating animal names among healthy young adults: result of stride velocity or effect of attentiondemanding task? Gait Posture 2008, 27:138-43

39. Hausdorff JM: Gait variability: methods, modeling and meaning. J Neuroeng Rehabil 2005, 2:19.

40. Morris ME, lansek R, Matyas TA, Summers JJ: Ability to modulate walking cadence remains intact in Parkinson's disease. J Neurol Neurosurg Psychiatry 1994, 57:1532-4.

41. Martin MY, Bradley LA, Alexander RW, Alarcón GS, Triana-Alexander M, Aaron LA, Alberts KR: Coping strategies predict disability in patients with fibromyalgia. Pain 1996, 68:45-53.

42. Sheridan PL, Solomont J, Kowall N, Hausdorff JM: Influence of executive function on locomotor function: divided attention increases gait variability in Alzheimer's disease. J Am Geriatr Soc 2003, 51:1633-7.

43. Goubert L, Crombez G, Van Damme S, Vlaeyen JWS, Bijttebier P, Roelofs J: Confirmatory factor analysis of the Tampa Scale for Kinesiophobia: Invariant two-factor model across low back pain patients and fibromyalgia patients. Clin J pain 2004, 20:103-10.

44. Keefe FJ, Hill RW: An objective approach to quantifying pain behavior and gait patterns in low back pain patients. Pain 1985, 21:153-61.

45. van Koulil S, Effting M, Kraaimaat FW, van Lankveld W, van Helmond T, Cats H, van Riel PLCM, de Jong AJL, Haverman JF, Evers AWM: Cognitivebehavioural therapies and exercise programmes for patients with fibromyalgia: state of the art and future directions. Ann Rheum Dis 2007, 66:571-81.

46. Vaillant J, Guinot M, Auvinet B, Chaleil D, Dumolard A, Favre-Juvin A, Wuyam $B$, Juvin R: Effects of rehabilitation exercise training on functional balance tests and gait markers in patients with fibromyalgia. Physiotherapie 2011, 97(Supplement S1).

47. Paleacu D, Shutzman A, Giladi N, Herman T, Simon ES, Hausdorff JM: Effects of pharmacological therapy on gait and cognitive function in depressed patients. Clin neuropharmacol 2007, 30:63-71.

48. Mork PJ, Vasseljen O, and Nilsen TIL: Association between physical exercise, body mass index, and risk of fibromyalgia: longitudinal data from the Norvegian Nord-Trondelag health study. Arthritis Care \& Research 2010, 62:611-7.

\section{Pre-publication history}

The pre-publication history for this paper can be accessed here:

http://www.biomedcentral.com/1471-2474/12/258/prepub doi:10.1186/1471-2474-12-258

Cite this article as: Auvinet et al:: The interest of gait markers in the identification of subgroups among fibromyalgia patients. $B M C$ Musculoskeletal Disorders 2011 12:258.

\section{Submit your next manuscript to BioMed Central and take full advantage of:}

- Convenient online submission

- Thorough peer review

- No space constraints or color figure charges

- Immediate publication on acceptance

- Inclusion in PubMed, CAS, Scopus and Google Scholar

- Research which is freely available for redistribution

Submit your manuscript at www.biomedcentral.com/submit
Biomed Central 